\title{
No. XXII.-TERRESTRIAL ISOPODA, PARTICULARLY CONSIDERED IN RELATION TO THE DISTRIBUTION OF THE SOUTHERN INDO- PACIFIC SPECIES*.
}

\author{
By the late Dr G. Budde-Lund, Copenhagen. \\ (Communicated by Prof. J. Stanley Gardiner, M.A., F.R.S., F.L.S.; \\ Revised by Rev. T. R. R. Stebbing, M.A., F.R.S.)
}

(Plates 20-22.)

Read 20th June, 1912.

THE collections of Terrestrial Isopoda obtained from the islands of the Indian Ocean during the Sealark Expedition were made in the following localities: Chagos Islands, Cargados, Coetivy, Farquhar, Amirante Islands, and Seychelles.

While collections from the Seychelles Islands have been gathered by different persons, until now no investigation appears to have been made with regard to these animals living in the five first-mentioned localities. Consequently I have set forth here all that I have found belonging to the Isopod-fauna of these islands.

From the Chagos Islands the Sealark Expedition has brought back the following six species :

Nagara nana Budde-Lund, a species found in Madagascar; I have also seen specimens from Java and Sumatra.

Metoponorthus pruinosus (Brandt); this cosmopolitan species is taken in all the islands and in great number.

Alloniscus pigmentatus Budde-Lund; this species, first found in Madagascar, was later taken in many localities on the coasts of the Indian Ocean.

Setaphora pilosa, n. sp. This new species, which I describe below, is related to many described and hitherto undescribed species found in the Indian islands.

Olibrinus pigmentatus, n. sp. This new species, which I describe below together with two other new species, one from Djibouti, the other from the Malay Peninsula, belonging to the same new genus, points as well as the above-mentioned species to the affinity of the fauna with the fauna of the East Indies.

Spherillo parvus Budde-Lund, from Madagascar, is spread over the islands of the Indian Ocean.

* All the footnotes are by the Revisor.

SECOND SERIES-ZOOLOGY, VOL. XV. 
From the Cargados Islands the expedition brought back three species :

Angara lenta Budde-Lund. This species is known from most countries around the Mediterranean Sea; perhaps it is of cosmopolitan distribution.

Metoponorthus* pruinosus (Brandt), a cosmopolitan species.

Trichorhina minutissima, n. sp. Though this species is a new one, I dare not consider it as peculiar to these islands. The species of the genus Trichorhina are very tiny animals, and, inhabiting moss and mouldering vegetable refuse, easily escape collectors. Further, because of their white colour they are easily confounded with the young of other species. This species, I think, has a wider diffusion.

From Coetivy Island the expedition has brought back four species :

Angara lenta Budde-Lund.

Metoponorthus pruinosus Brandt.

Setaphora suarezi (Dollfus).

Spherillo parvus Budde-Lund.

The two last species have probably entered from Madagascar; the other two are nearly cosmopolitan.

In the island Farquhar were collected during the expedition the following six species:

Metoponorthus pruinosus (Brandt).

Agnara madagascariensis Budde-Lund.

Alloniscus pigmentatus Budde-Lund.

Aphiloscia annulicornis Budde-Lund.

Setaphora suarezi (Dollfus).

Bethalus simplex (Dollfus).

These species are not peculiar to Farquhar. They all inhabit Madagascar and some extend into East Africa.

From the Amirante Islands are also presented four species, all cosmopolitan :

Angara lenta Budde-Lund.

Cubaris murina Brandt.

Metoponorthus pruinosus (Brandt).

Spherillo parvus Budde-Lund.

Most rich and interesting is the fauna of the Seychelles Archipelago. The first species of terrestrial Isopods brought home by Prof. Möbius were only two, Cubaris murina Brandt and Spherillo parvus (Budde-Lund (1) p. 28 and p. 25). In the year 1893 M. A. Dollfus $†$ gave a catalogue of the species collected by M. Charles Alluaud, in all seven species. Besides the two species above named, he had brought home Metoponorthus pruinosus (Brandt), Alloniscus pallidulus Budde-Lund $\ddagger$ Pseudophiloscia angustissima Budde-Lund $\$$ Ligia exotica Roux, and Tylos minor Dollfus.

* That Metoponorthus, Budde-Lund, 1879, is a synonym of Porcellionides, Miers, 1877, is explained by Stebbing in "Records of the Indian Museum," vol. vi., pt. 4, p. 188, Sept. 1911.

+ Bull. soc. zool. Fr. vol. xviii. p. 186. Paris, 1893.

$\ddagger$ Anomaloniscus ovatus Dollfus.

$\S$ On p. 372 the species by Dollfus supposed to be Philoscia mina, Budde-Lund, is stated to be P. lateralis. 
Subsequent collectors and the Sealark expedition have obtained twelve species new to the fauna of Seychelles, and so the total number of the species found in the Archipelago is to-day twenty:

1. Spherillo parvus (Budde-Lund) M.

2. " peltatus Budde-Lund S.

3. „ maculosus Budde-Lund S.

4. Pseudophiloscia lateralis, n. sp. S.

5. " angustissima, n. sp. S.

6. Mahehia maculata, n. sp. S.

7. ", laticauda, n. sp. S.

8. ", bicornis, n. sp. S.

9. Cubaris murina Brandt C.

10. Pagana dimorpha (Dollfus) M.

11. Metoponorthus pruinosus (Brandt) C.

12. Nagara cristata (Dollfus) C.

13. „, nana Budde-Lund M.

14. Alloniscus pallidulus Budde-Lund M.

15. " pigmentatus Budde-Lund M.

16. Setaphora ovata, n. sp. S.

17. ", pallidemaculata, n. sp. S.

18. Aphiloscia annulicornis (Budde-Lund) M.

19. Ligia exotica Roux C.

20. Tylos minor Dollfus S.

Among these twenty species the half, 10 species, above marked with " $\mathrm{S}$," are peculiar to Seychelles; four species marked with " $\mathrm{C}$ " may be considered as cosmopolitan ; and six species marked with an "M" are common to these islands and Madagascar, being regarded as certainly introduced from that country.

It is not only the large number of peculiar species that gives to the fauna of Seychelles its particular stamp. It is rather the character of the species; thus there are three species of a new genus, Mahehia, of which no representative is known from anywhere else, and two species of a genus Pseudophiloscia, of which species are not found nearer than New Zealand, Upolu, Chile. The species of Spherillo and Setaphora only point to a connection with the Australasian Islands, while no form points to any connection with Africa, Madagascar being excepted.

From Mauritius are known the following ten species :

1. Sunniva minor Budde-Lund.

2. Spherillo testudinalis (Budde-Lund). 
3. Spherillo collaris Budde-Lund.

4. " parvus Budde-Lund.

5. Angara lerta Budde-Lund.

6. Pagana dimorpha Dollfus.

7. $\quad$ maculosa Budde-Lund.

8. " fissifrons Budde-Lund.

9. Metoponorthus pruinosus Brandt.

10. Aphiloscia annulicornis Budde-Lund.

Four species, nos. 1, 3, 6, 7, are known only from Mauritius. The other six species are found in Madagascar and the East Indies.

In my paper on the Isopoda from Madagascar (Budde-Lund (5)) I have recorded 32 species as inhabiting that large island.

L. A. Borradaile in his paper on the Land Crustaceans in the Fauna: Maldives and Laccadives, vol. i., p. 28, has recorded six species from those islands :

Ligia exotica Roux.

Porcellio maldivensis Borradaile=Agnara madagascariensis Budde-Lund?

Alloniscus maldivensis Borradaile =Alloniscus pigmentatus Budde-Lund?

Philoscia gracilis Budde-Lund ? = Setaphora sp.

$$
\text { ,"sp. }
$$

Cubaris murina Brandt.

The descriptions of the new species given by Borradaile are very superficial* and apply to many different species, being without essential characters.

Besides the Isopoda collected by the Sealark Expedition $\mathrm{Mr}$ Thomasset during a sojourn at the Aldabra Islands has taken a new species, Tura angusta, which I on this occasion describe together with the other species of this genus.

This small collection of the Isopod-Fauna confirms the conclusion I have drawn (Budde-Lund (2), p. 40) that the Seychelles Islands and East Madagascar are remnants of a submerged continent, which occupied a large part of the globe, now to a great extent covered by sea.

While the Seychelles Islands, East Madagascar, Mauritius and Bourbon mark the occidental outline of this submerged continent, the west coast of South America is its oriental outline, confined by the Andes mountains; of the countries between these two outlines some of the East Indian Islands, the Malay Peninsula, East Australia, New Zealand and the Pacific Islands are remnants. In those times certainly North and South America were separated, and several of the West Indian Islands (Cuba, Porto Rico and Jamaica) were belonging to a submerged continent.

* It should be remembered that Borradaile's paper was published in 1901 before the dawn of that new era, in which, as a comparison of Herr Budde-Lund's earlier and later writings clearly testifies, the terrestrial Isopoda have been enabled to claim a far more searching analysis than in earlier times they were accustomed to receive. 
Another more recent connection has existed between West Africa and South America and between South Africa and West Australia.

\section{Familia Oniscidæ.}

Onisci Budde-Lund (1), p. 13, 1885.

Subfamilia Spherilloninæ Budde-Lund (2), p. 41, 1904.

Included in this subfamily are a number of genera, Spherillo, Sunniva, Sä̈djahus, Scleropactes, Suarezia, Arhina, Pseudophiloscia, Ambounia, Pseudarmadillo, Acanthoniscus and Anaphiloscia.

I have earlier given the reasons for joining these genera in a subfamily. Of the above-named genera the Sealark Expedition has brought back species of the genera Spherillo, Pseudophiloscia, and a new genus Mahehia.

Genus Spherillo Budde-Lund (2), p. 51 Dana.

(U. S. Expl. Exp., vol. xiii., p. 719, 1853.)

Five species of this genus are found in the Islands of the Indian Ocean. Two of them are only known from Seychelles, one only from Mauritius, the other species are of wide distribution eastwards.

\section{Spherillo parvus.}

Armadillo parvus Budde-Lund (1), p. 25.

Spherillo parvus Budde-Lund (2), p. 91 ; B.-L. (5), p. 270 . Taf. 12, fig. 30-37.

Distribution. Seychelles Archipelago: Mahé, 1600 feet, many specimens; Mt. Harrison at an altitude of 2000 feet; Trois frères, 1500 feet, 17 Nov. 1905 : Silhouette a single specimen: Praslin on dry hills, five specimens. Desroches, very abundant. Coetivy, seven specimens. Chagos Archipelago at Coin, Peros, and at Egmont, many specimens.

This species was taken many years ago in Seychelles by Prof. Möbius; later M. Ch. Alluaud found it at Mahé, at Praslin and in Madagascar; also specimens were obtained from Mauritius. In the British Museum, London, are preserved specimens from CocosKeeling Island, taken in July 1902 by Mr Wood-Jones.

2. Spherillo purpurascens, n. sp.

Cubaris officinalis Stebbing, Willey's Zool. Results, 1900, p. 655.

Oculi mediocres, ocelli magni, numero 13.

Antennarum articulus 1 flagelli duplo brevior quam articulus 2.

Trunci segmentum 1 margine laterali crasso, per totam longitudinem sulcato, post inaequaliter sulcato: lacinia exterior multo major quam lacinia interior; margo posterior utrinque leviter incurvus. Segmentum 2 epimeris ante fissis, lacinia interiore brevi, obtusa; pronotum fere quartæ parti dorsi longitudine æquale; margo posterior utrinque levissime incurvus. 
Uropodum endopoditum apicem telsi non attingens ; exopoditum minutum, non, nisi desuper, visibile, in fossa laterali scapi positum.

Color fusco et flavo variegatus ; interdum fuscus maculis pallidis notatus.

Lg. $13 \mathrm{~mm}$.

Distribution: Isle of Pines ; South of New Caledonia

[Spherillo purpurascens is certainly a new species. In the manuscript of the late Mr Budde-Lund, however, this was not expressly stated.]

3. Spherillo collaris.

Spherillo collaris Budde-Lund (2), p. 60, 1904.

Mauritius (Mus. Paris).

4. Spherillo peltatus.

Spherillo peltatus Budde-Lund (2), p. 78, 1904.

Seychelles Archipelago (Mus. Hamburg).

5. Spherillo testudinalis.

Armadillo testudinalis Budde-Lund (1), p. 29, 1885.

Spherillo $\quad$ " Budde-Lund (2), p. 80, 1904.

Mauritius (Mus. Paris). This species is spread over the greatest part of the Asiatic and Australian islands; also found in Madagascar.

6. Spherillo maculosus.

Spherillo maculosus Budde-Lund (2), p. 80, 1904.

Seychelles Archipelago, Mahé (Mus. Hamburg).

Genus Pseudophiloscia Budde-Lund (2), p. 42, 1904.

The discovery in the Seychelles of the two new species described below is very interesting and corroborates my hypothesis concerning ancient continental lands.

I have before recorded three species in this genus: Pseudophiloscia gracilis (BuddeLund) (1), p. 220 and (2), p. 42 from Upolu Island; Pseudophiloscia fragilis Budde-Lund (2), p. 43 from New Zealand; Pseudophiloscia inflexa Budde-Lund (2), p. 43 from Chili. I should also think the Paraphiloscia stenosoma Stebbing*, from New Britain, to be a Pseudophiloscia $\dagger$, whereas the Philoscia gracilis Borradailef from the Maldives is certainly not identical with Ph. gracilis Budde-Lund but rather a Setaphora.

7. Pseudophiloscia lateralis, n. sp. (Plate 20, figs. 1-6.)

Philoscia mina Dollfus (1), p. 188 (neque Budde-Lund), 1893.

* Stebbing, Willey's Zool. Results, 1900, p. 648.

$\dagger$ In agreement with this opinion it should be conceded that Pseudophiloscia Budde-Lund, 1904, becomes a synonym of Paraphiloscia, Stebbing, 1900, and that Budde-Lund's three species above named should consequently be transferred to the latter generic name.

$\ddagger$ L. A. Borradaile, Land Crustaceans, Gardiner's Fauna Maldives and Laccadives, i. p. 98. 
Superficies sublævis; nitida, setis minutissimis sparse munita.

Oculi magni, angulos anticos capitis utrinque occupantes; ocelli numerosi, densissime congregati, numero plus quam viginti.

Antennæ fere tres quartas partes corporis longitudine æquantes; scapi articulus 2 paulo longior et crassior quam articulus 3 ; articulus 4 plus duplo longior quam articulus 3 , articulus 5 quarta parte longior quam articulus 4 . Flagellum paulo longius quam articulus 5 scapi, articulus 1 longior quam articuli 2 et 3 subæquales simul sumpti.

Maxillæ prioris paris lamina exterior dentibus 4 (secundo ceteris multo minore) +6 (omnibus apice fissis, subæqualibus).

Frons ante non marginata. Epistoma linea transversa in medio leviter procurva.

Trunci segmenta 1, 2 margine posteriore curvato, segmenta 3, 4 margine posteriore subrecto, segmenta 5, 6 leviter segmentum 7 profunde in medio incurva; anguli postici segmentorum 1-4 rotundate obtusi, segmentorum 5-6 subrecti, segmenti 7 rotundate truncati. Segmentum 2 pronoto quarta parte dorsi vix longiore, ægre discreto, utrinque angulos priores laterales segmenti occupante.

Cauda trunco abrupte angustior; omnia segmenta, præsertim segmenta 4 et 5 magna.

Telsum triangulum, lateribus leviter incurvis, post late rotundatum.

Uropodum scapus multo longior quam latior, latere exteriore profunde canaliculato; processus endopoditi non brevior quam scapus; exopoditum duplo longius quam scapus; endopoditum gracile, paulo longius quam scapus.

Color fuscus, in medio trunco maculis et striis oblongis crebratus; segmenta trunci macula laterali rotunda albida, etiam anguli postici segmentorum perlucente albidi.

Long. $8 \mathrm{~mm}$. Lat. $3.5 \mathrm{~mm}$.

Distribution. Seychelles Archipelago: many specimens of this species were obtained during the Sealark expedition: Montagne Alphonse, Cascade, 1800 feet; summit Mt. Sebert; Silhouette. In the Museum at Hamburg are preserved specimens taken by . Dr A. Brauer in Mahé, 2-8 May, 1901. It had already been taken in the year 1892 by M. Ch. Alluaud at Mahé, Marianne and Praslin; Dollfus referred it wrongly to Philoscia mina Budde-Lund. I have seen the original specimens in the Museum at Paris.

8. Pseudophiloscia angustissima, n. sp. (Plate 20, figs. 7-10.)

Superficies lævis, nitidissima.

Oculi mediocres, angulos anticos capitis utrinque occupantes; ocelli minuti, subconfluentes.

Antennæ duas corporis partes longitudine subæquantes; scapi articulus 2 non longior quam articulus 3 , articulus 4 fere duplo longior quam articulus 3 , articulus 5 quarta parte longior quam articulus 4. Flagellum scapi articulo 5 longitudine æquale.

Maxillæ prioris paris lamina exterior dentibus 4 (dens primus posterior longissimus, 2 longus, 3 et 4 parvi) +6 (dens 1 longus, 5 parvus, ceteri mediocres, omnes acuti, integri). 
Frons ante non marginata. Epistoma linea transversa recta, infra inter antennulas tuberculo ovali, minuto.

Trunci segmenta 1, 2, 3 margine posteriore curvato, segmentum 4 subtransversum, segmenta 5, 6 levissime, segmentum 7 fortius medio incurvo; anguli postici segmentorum 1, 2, 3 late rotundate obtusi, anguli segmentorum 4, 5, 6 rotundate subrecti, segmenti 7 acutiores.

Cauda trunco abrupte angustior; segmenta angusta post sensim longiora; $1,2,3$ breviora, 4, 5 longa; epimera inflexa, brevia, non post retroducta.

Telsum plus duplo latius quam longius; basis brevis, transverse rectangula; apex brevis, triangulus, lateribus levissime incurvis, post obtusior.

Uropodum scapus manifesto longior quam latior, latere exteriore late et profunde per longitudinem canaliculato; processus endopoditi nonnihil brevior quam scapus.

Color e fusco griseus maculis et striis crebris albescentibus ornatus.

Long. $5 \mathrm{~mm}$. Lat. $1.5 \mathrm{~mm}$.

Distribution. Seychelles Archipelago: Mahé; Mt Alphonse, 3 Dec. 1905, 3 specimens. The Museum at Hamburg possesses 7 specimens taken by Dr A. Brauer, 2 May, 1901.

\section{Pseudophiloscia brevicornis, n. sp. (Plate 20, figs. 11-16.)}

Superficies minute et densius hirsuta, nitida.

Oculi minores; ocelli pauci, numero 9-10.

Antennæ breviores, tertiam partem corporis longitudine vix æquantes; scapi articuli ad apicem versus gradatim longiores; flagellum scapi articulo 5 longitudine subæquale, articulo primo ceteris longiore.

Maxillæ prioris paris lamina exterior dentibus 2 (dens prior postremus longissimus, validus) +4 (dens 2 ceteris subæqualibus nonnihil longior, omnes acuti, integri).

Frons ante non marginata. Epistoma linea transversa tenui, recta, infra inter antennulas transverse elevatius.

Trunci segmenta 1-4 margine posteriore curvato, segmentum 5 margine posteriore subtransverso, segmenta 6 et 7 margine posteriore in medio leviter incurvo; anguli postici segmentorum $1-5$ rotundate obtusi, anguli postici segmenti 6 subrecti, anguli postici segmenti 7 acutissimi, apice retroducto.

Cauda trunco non abrupte angustior; segmenta $1-5$ lata, subæque longa, epimeris minutis, adpressis.

Telsum plus duplo latius quam longius, post rotundate triangulum.

Uropodum scapus paulo longior quam latior, latere exteriore late et profunde per longitudinem sulcato; processus endopoditi nonnihil brevior quam scapus. Exopoditum semiplice longius quam scapus, apice stylis longissimis ornatum. Endopoditum vix longius quam scapus, apice stylis longis ornatum.

Color flavus crebre fuscomaculatus, in epimeris fuscis macula rotunda flava; antennæ albescentes. 
Long. $7.5 \mathrm{~mm}$. Lat. $1.5 \mathrm{~mm}$.

Distribution. New Zealand, Auckland. I have examined a few specimens sent by Mr Suter to the Museum at Hamburg under the name Oniscus punctatus Thoms. This adds a second species to the New Zealand fauna and a sixth species to the genus, which ranges from Seychelles to Chili.

\section{Mahehia, nov. gen.}

Antennarum flagellum 2-articulatum.

Pleuræ capitis concretæ; linea marginalis verticalis utrinque ante oculos producta, marginem frontalem attingens.

Trunci segmentum primum epimeris integris, infra dente longo, acuto instructis.

Pleopodes omnium parium tracheis instructi.

Telsum triangulum, apice truncato aut acuto.

Uropodes longi, producti ; exopoditum longum.

Utrum adsit necne folliculus abdominalis sive marsupium, nescio.

10. Mahehia maculata, n. sp. (Plate 20, figs. 17-25.)

Superficies capitis et segmentorum priorum trunci maxime segmenti primi subtiliter et sparse granulata, praesertim in lateribus, post sublævis, nitidissima.

Oculi magni, ocelli numero c. 23.

Antennæ longissimæ, fere $\frac{5}{6}$ corporis longitudinem superantes; scapi articulus 1 , brevissimus, articulus 2 brevis, crassior, articulus 3 fere triplo longior quam articulus 2, articulus 4 paulo longior quam articulus 3 et gracilior, articulus 5 nonnihil longior quam articulus 4 gracillimus. Flagellum gracillimum, duas partes scapi articuli 5 longitudine æquans, articulo priore plus duplo breviore quam articulo altero; articulus alter seta apicali instructus.

Frons ante marginata, utrinque lobata; lobi laterales minores, rotundati, linea marginalis crassior in medio leviter procurva; frons utrinque intra lobos laterales tuberculo oblongo, parvo, obliquo instructa. Epistoma convexum, in medio carinis duabus curvatis se attingentibus, a margine frontali ad clypeum ductis, munitum. Tubercula antennaria majora, acutiora, exteriora.

Trunci segmentum 1 post marginem anteriorem sulco profundo per transversum ducto; margo posterior utrinque profunde incurvus, angulis lateralibus acutissimis, retroductis; epimerum infra procul a margine laterali dente longo, acuto instructum. Segmentum 2 pronoto magno, plus tertiam partem dorsi occupante; margo posterior subtransversus, angulis lateralibus rotundate obtusis.

Caudæ segmenta $3,4,5$, epimeris magnis; epimera segmenti 5 lateribus interioribus parallelis, telso paulo brevioribus. Pleopodes primi paris in utroque sexu parvi, inter se longe distantes.

Telsum triangulum; paulo, fere quinta parte latius quam longius, lateribus leviter incurvis, apice obtusiore, supra per medium ad longitudinem impressum. 
Uropodum exopoditum plus quam dimidio longius quam scapus.

Color e nigro fuscus ; in trunci segmentis maculæ senæ aut octonæ parvæ rotundæ in seriem transversam, in caudæ segmentis maculæ quaternæ aut senæ sæpe evanidæ positæ; antennæ nigræ.

Long. $19 \mathrm{~mm}$. Lat. $8 \mathrm{~mm}$.

Distribution. Seychelles Archipelago. I have seen four specimens of this species from Mahé. Two specimens were taken during the Sealark expedition, 3 December 1905 ; the other two, preserved in the Museum at Hamburg, were found by Dr Brauer, 2 May 1900.

11. Mahehia laticauda, n. sp. (Plate 20, fig. 26 ; plate 21, fig. 1-6.)

Superficies trunci medii sparse granulata, epimerorum segmentorum trunci et cauda dense squamata.

Oculi majores vel magni ; ocelli numero c. 14, posteriores ceteris majores.

Antennæ graciles, longiores, dimidio corpore paulo longiores; scapi articulus 1 brevissimus, articulus 2 brevis, subglobosus, articulus 3 duplo longior quam articulus 2, articulus 4 tertia parte vel plus longior quam articulus 3 , articulus 5 quarta parte longior quam articulus 4, gracilis. Flagellum gracile, paulo longius quam dimidium articuli $\mathbf{5}$ scapi, articulo priore plus duplo breviore quam articulo altero, seta apicali instructo.

Frons ante marginata, utrinque lobata, lobi laterales minores, oblique rotundati, linea marginalis ad mediam frontem fortius procurva. Epistoma in medio convexum, supra eminentia semicirculata marginata infra et ad latera excavatum.

Trunci segmentum 1 sulco transverso collari minus profundo; margo posterior utrinque levius incurvus, angulis lateralibus acutis, non retroductis; epimerum infra procul a margine laterali dente longo, acuto instructum. Segmentum ź pronoto magno, margo posterior levissime utrinque incurvus. Segmenta 2-7 epimeris magnis, tetragonis, paulum obliquis, angulis lateralibus subrectis.

Caudæ segmenta 3,4 epimeris permagnis; epimera segmenti 5 valida, oblique tetragona, lateribus interioribus subparallelis.

Telsum trapezoidale vel triangulum apice late truncato, paululo (septima parte) latius quam longius.

Uropodum scapus dimidio longior quam latior, exopodito pæne dimidio longior.

Subunicolor, grisea, sæpe decolorate pallida; antennarum articulus 5 scapi parte apicali albida.

Long. $13 \mathrm{~mm}$. Lat. $7.5 \mathrm{~mm}$.

Distribution. Seychelles Archipelago: Mahé; many specimens taken on "Montagne Alphonse, Cascade, 1800 feet," in the days 30 Nov., 2 Dec., 4 Dec. 1905 : Praslin, three specimens from 1000 feet under dead palm leaves.

12. Mahehia bicornis, n. sp. (Plate 21, figs. 7-11.)

Tota superficies densius et scabrius maxime ad latera et in epimeris trunci granulata. 
Oculi magni, semiglobosi ; ocelli numero c. $22-23$.

Antennæ longissimæ; scapi articuli et flagellum ut in $M$. maculata.

Frons ante marginata et utrinque lobata; lobi laterales longiores et angustiores, subtrianguli, apicibus obtusis, linea marginalis frontalis in medio profundius emarginata, ut media frons bicornis fiat. Epistoma medium triangulis elevatis, superiore et inferiore se in medio attingentibus, convexum. Clypeus magnus, declivis, non lobatus.

Trunci segmentum 1 sulco anteriore collari tenui; margo posterior utrinque profundius incurvus, angulis lateralibus acutissimis, non retroductis; epimerum infra dente longo, gracili, acuto. Segmentum 2 pronoto magno. Segmenta 2-7 margine posteriore utrinque levissime incurvo, angulis lateralibus post sensim acutioribus.

Caudæ segmenta 3,4 epimeris magnis; epimera segmenti 5 epimeris precedentibus angustiora, acute triangula, telso paulo breviora, lateribus interioribus subparallelis.

Telsum triangulum, ejusdem longitudinis atque latitudinis, lateribus levissime incurvis, apice obtusiore, supra per medium ad longitudinem leviter sulcatum.

Uropodum exopoditum multo longius quam scapus.

Color in medio corpore e nigro fuscus, in epimeris trunci caudæque flavus ; antennæ flavæ vel albidæ; color interdum pulchre purpureus, lateribus anguste albidis.

Long. $16 \mathrm{~mm}$. Lat. $8 \mathrm{~mm}$.

Distribution. Seychelles Archipelago: Mahé, one specimen from mountain forest, Nov. 1905 : Silhouette, five specimens. I have also had many specimens for examination, taken the 2 May 1901 by Dr A. Brauer and preserved in the Museum, Hamburg.

\section{Subfamilia Oniscinæ. Budde-Lund (1), p. 75.}

This subfamily contains the largest number of the known forms of terrestrial Isopods. In nearly sixty genera are contained about one thousand species. Authors have produced an abundance of new species on very superficial descriptions. These are very often quite incorrectly placed, because the external characters on which the affinities are founded are often without any value.

I have lately in a paper $((7)$, p. 8$)$ given hints, which should certainly lead to the understanding of the affinities between the numerous genera. By later examinations of the given characters and of other new ones, I have reached so far as to think that I can give a survey of the genera, not only useful for determination but also in the main points in accord with a natural system.

Subgenus Cubaris Brandt, Bull. Soc. Nat. Moscou, vol. vi. p. 189, 1833.

$$
\text { Budde-Lund (6), p. 54, } 1909 .
$$

13. Cubaris murina.

Cubaris murina Brandt, Conspectus, p. 28, 1833.

Armadillo murinus Budde-Lund (1), p. 27 ; (2), p. 119 . Tab. X. figs. 20-22.

Cubaris murina Budde-Lund (6), p. 54 . 
Seychelles Archipelago: Silhouette and Curieuse Island very abundant: Praslin, Cascade, $700 \mathrm{ft}$. ; Mahé, on Mt. Harrison, alt. $2000 \mathrm{ft}$.; Trois Frères, $1500 \mathrm{ft}$., the $17 \mathrm{th}$ Nov. 1905 : Desroches, very common in sand hills. Already Prof. Möbius had taken this species in the Seychelles, also M. Ch. Alluaud in Mahé and Marianne (sec. Dollf.). Specimens from Mahé are also preserved at the Museum, Hamburg, found by $\mathrm{Dr} A$. Brauer.

Concerning the wide distribution of this species, consult the l.c., 2, p. 119, where all the hitherto known localities are mentioned. I can add that specimens have been obtained from Cocos-Keeling Island ( $\mathrm{Mr}$ Wood-Jones in Museum, London).

Genus Porcellio Latreille, Hist. Nat. Crust. et Ins., vol. vii., p. 45, 1804. Budde-Lund (5), p. 280.

Subgenus Tura Budde-Lund (5), p. 282, 1908.

During his stay at the Aldabra Islands in 1907, Mr H. P. Thomasset took several specimens of a little new species belonging to this genus. Till then I knew only one species, also from the Aldabra Islands, taken by Prof. Voeltzkow, who had secured it also in N. Madagascar. Subsequently I have seen two more new species from Abyssinia; later on I give a review of all these nearly related species.

\section{Tura angusta, n. sp.}

Superficies squamis minutissimis dense obtecta.

Frons cum epistomate superiore leviter producta, crista squamarum minutissimarum ante marginata ; processus laterales minores, late rotundati.

Trunci segmenta 1, 2, 3 margine posteriore curvato, segmentum 4 margine posteriore subrecto, segmenta 5, 6,7 margine posteriore in medio fortius incurvo; anguli laterales posteriores segmentorum 1, 2, 3 late rotundate obtusi, segmenti 4 rotundate subrecti, segmentorum 5, 6, 7 acutiores.

Caudæ epimera segmentorum 3, 4, 5 longiora, angusta, acuta, subadpressa.

Telsum breve, triangulum, epimera segmenti 5 satis superans, lateribus fortiter incurvis, apice acutissimo.

Color e fusco brunneus, obscure pallide maculatus.

Long. $4.5 \mathrm{~mm}$. Lat. $2 \mathrm{~mm}$.

Distribution. Aldabra : several specimens (H. P. Thomasset).

\section{Tura testacea.}

Tura testacea Budde-Lund (5), p. 282. Tab. 14, fig. 1-14, 1908.

Superficies squamis minutis dense obtecta.

Frons media cum superiore epistomate leviter bulbose producta, ante obscure marginata; processus laterales rotundate obtusi.

Trunci segmenta 1, 2, 3 margine posteriore curvato, segmenta 4-7 margine posteriore in medio leviter incurvo; anguli laterales posteriores segmentorum $1,2,3$ 
late rotundate obtusi, segmenti 4 rotundate subrecti, segmentorum $5,6,7$ acutiores, subrecti.

Caudæ epimera segmentorum $3,4,5$ breviora, latiora, distantia.

Telsum breve, triangulum, duplo latius quam longius, epimera segmenti 5 nonnihil superans, lateribus leviter incurvis, apice acutiore.

Unicolor, testaceus.

Long. $6-7 \mathrm{~mm}$. Lat. $3-3 \cdot 7 \mathrm{~mm}$.

Distribution. Aldabra. Majunga in W. Madagascar (Museum, Berlin).

16. Tura albipennis, n. sp.

Superficies squamis minutissimis sparsius obtecta.

Frons ante leviter producta, ab epistomate linea squamarum minus manifesto discreta; processus laterales majores, oblique rotundati.

Trunci segmentum 1 margine posteriore curvato; segmenta $2-3$ margine posteriore subrecto vel levissime curvato; segmenta $4-7$ margine posteriore post sensim in medio magis incurvo; anguli laterales posteriores segmentorum 1, 2, 3 rotundate obtusi, segmentorum $4-7$ acuti.

Caudæ segmentorum 3, 4, 5 epimera latiora, acuta, distantia.

Telsum late triangulum, epimera segmenti 5 nonnihil superans, lateribus leviter incurvis, apice acuto. latius.

Uropodum scapus telso multo brevior; exopoditum breve, vix duplo longius quam

Unicolor e flavo brunneus; antennæ albæ.

Long. $7 \mathrm{~mm}$. Lat. $4 \mathrm{~mm}$.

Distribution. Abyssinia, near Harrar and at Dire-Danah, 5 specimens taken by Herr E. Wache preserved in the Museum, Hamburg.

17. Tura laticauda, n. sp.

Superficies squamis crassioribus densissime obtecta.

Antennarum scapi articuli ad longitudinem sulcati.

Frons ante cum superiore epistomate fortius producta et marginata; processus laterales majores, prominentes, latere interiore rotundato ; epistoma convexum, infra linea transversa obscuriore in medio procurva munitum.

Trunci segmenta 1, 2 margine posteriore curvato, segmentum 3 margine posteriore subrecto, segmenta 4-7 margine posteriore post sensim in medio magis incurvo; anguli laterales posteriores segmentorum 1,2 late rotundati, segmenti 3 rotundate subrecti, segmentorum $4-7$ post gradatim acutiores.

Caudæ epimera segmentorum 3, 4, 5 breviora, latiora, distantia.

Telsum breve, triangulum, epimera segmenti 5 vix superans, lateribus fortius incurvis, apice acuto.

Unicolor, e testaceo fuscus. 
Long. 5.5-6.5 mm. Lat. $3 \cdot 2--3.5 \mathrm{~mm}$.

Distribution. Abyssinia, Rio Faressa (Mission du Bourg de Bozas de Feltner, Juin 1901, 4 specimens in Museum, Paris); Arussi Galla, at Aduabeba in "Hanasch Thal," 2 specimens (O. Neumann in Museum, Berlin).

Perhaps the following species belongs to this genus: Leptotrichus inquilinus Koelbel, Wasmann, Verzeichn. myrmecoph. u. termitoph. Arthrop. Berlin, 1894, p. 221 ; from Somaliland.

Subgenus Pagana Budde-Lund (5), p. 287, 1908.

\section{Pagana dimorpha.}

Metoponorthus dimorphus Dollfus, Mém. Soc. Zool. Fr. viii., p. 184, 1895.

Pagana dimorpha Budde-Lund (5), p. 288, Taf. 15, fig. 1-10.

Seychelles Archipelago. I have only seen the rudiments of a single specimen from "Baie Praslin, 1876," taken by M. de l'Isle (Mus. Paris). The species is also found at Réunion and Mauritius.

The other two known species of the genus, Pagana maculos $\alpha$, Budde-Lund (5), p. 288, and P. fissifrons, Budde-Lund (5), p. 289, are found only in Mauritius.

Subgenus Angara Budde-Lund (4), p. 5, 1908.

\section{Angara lenta.}

Lyprobius lentus Budde-Lund (1), p. 230.

Leptotrichus lentus Dollfus, Mém. Soc. Zool. Fr., ix. 542, 1896.

Angara lenta Budde-Lund (4), p. 7, Tab. 1, fig. 1-16.

The expedition has brought back specimens from the following localities: Cargados Islands, 5 specimens: Coetivy Island, 1 specimen: Desroches Atoll, 5 specimens.

This species seems to be cosmopolitan. I have seen specimens from Italy, Algeria, Egypt, Greece at Mykenæ (17. III. 1905, Dr R. Schütt in Mus. Hamburg), Port au Prince (7. V. 1894, 1 specimen in Mus. Hamburg), Progreso (20. X. 1905, 1 specimen, physician C. G. Budde-Lund), Mauritius (Dr Emmery, in Mus. Paris), Chester (Mr Beresford).

\section{Subgenus Metoponorthus Budde-Lund 1879.}

20. Metoponorthus pruinosus.

Porcellio pruinosus Brandt, Consp., p. 19.

Metoponorthus pruinosus Budde-Lund (1), p. 169.

This cosmopolitan species, spread world-wide, inhabits most of the islands of the Indian Ocean. The Sealark Expedition brought back specimens from the following localities: Chagos Archipelago: Coin, Peros; Salomon; Egmont; Diego-Garcia. Cargados Archipelago, Siren Island, very abundant. Farquhar, $30 \mathrm{Sept.1905.} \mathrm{Amirante}$ Archipelago, Eagle Island, 17 Oct. 1905. Seychelles Archipelago: Praslin; Mahé, Trois Frères, at an altitude of 1500 feet, and Mt. Harrison, 2000 feet; Bird Island. Coetivy. 
Subgenus Agnara Budde-Lund (5), p. 286, 1908.

21. Agnara madagascariensis.

Metoponorthus madagascariensis Budde-Lund (1), p. 189.

Agnara madagascariensis Budde-Lund (5), p. 286 . Taf. 14, figs. 48-54.

Farquhar Island, 30 Sept., 1905, a few specimens. This species seems not to be rare in North Madagascar.

Subgenus Bethalus Budde-Lund (6), p. 54, 1909.

22. Bethalus simplex (Plate 21, figs. 12-15).

Armadillo simplex Dollfus, Mém. Soc. Zool. Fr., viii. 180, text-fig. 1, 1895.

" " Budde-Lund (2), p. 132.

Distribution. Only two defective specimens were taken at Farquhar, 30 Sept. Certainly this species was imported from Madagascar, where found by M. Charles Alluaud on "Montagne d'Ambre."

To supplement the description given by Dollfus, l.c., I add a few essential characters; I also give drawings of other characteristic and instructive features.

Trunci segmentum 2 pronoto fere quintam partem dorsi æquante.

Pleopodes primi paris feminæ parvi, inter se longe distantes, area operculari subnulla.

Subgenus Nagara Budde-Lund (5), p. 284, 1908.

23. Nagara cristata.

Porcellio cristatus Dollfus, Notes fr. Leyden Mus., xi., 1891, Taf. v. fig. 2.

Nagara cristata Budde-Lund (5), p. 284, Taf. 14, figs. 27-39.

Seychelles Archipelago; eight specimens, all females, were collected at Mahé, at an altitude of 1600 feet.

This species is obtained also in Madagascar and seems to be widely spread over the countries within the warm zone. I have never seen a male specimen of this species.

\section{Nagara nana.}

Nagara nana Budde-Lund (5), p. 285, Taf. 14, figs. 40-47.

Seychelles Archipelago: one specimen was found at Praslin, dry hills; in the museum of Paris is also one specimen from Praslin. Chagos Archipelago: two specimens from Egmont, and four specimens from Coin, Peros.

Formerly, this species was only known from Madagascar; lately I have seen specimens from Java (Buitenzorg 24. II. and 2. III., Tjibodas 25. III. 04, Prof. K. Kraepelin in Mus. Hamburg), Singapore (Mus. Hamburg), Ceylon at Mahaväliganga (Dr G. Duncker 16. VIII. 09, in Mus. Hamburg), Formosa at Takao (Dec. 07, Mr H. Sauter in Mus. Hamburg). The species seems to be widely spread. 
Genus Trichorhina Budde-Lund (5), p. 293, 1908.

The species of Trichorhina are tiny Isopods, not exceeding $2-3 \mathrm{~mm}$. in length ; it is only natural, therefore, that they should often escape being taken by naturalists, most likely because of their likeness to young ones of other Isopods.

The Sealark Expedition brought back a rather large number of specimens of a species, which I class in this genus; while most of the characters of this species are confluent with the characters of the genus, given in the above-cited paper, the first pair of maxillæ presents a remarkable variation as to the teeth.

Such differences as to this pair of the trophi I have also observed in other species belonging to this group of Isopods, and I give a review of the species known by me, based on these relations.

Besides that the whole surface of the animal has a characteristic cover of tiny setaceous scales, found on all the species; an essential character may also be had from the mandibles, the inferior seta having only two or three branches and the interior lobe of the left mandible having a row of small papillæ (Plate 21, fig. 18).

T'ogether with the small species from the Siren Island, Cargados, brought back by the expedition, I describe below another new species from Mauritius. Many years ago I got a single small specimen of a species for which I could find no place in my system until now ; it was brought to Denmark on a sailing ship carrying a cargo of sugar.

\section{Conspectus SPECierum.}

Maxillæ prioris paris lamina exterior :

a. Dentibus $4+5$ (dentes 1, 2 apice fisso, dentes $3,4,5$ serrati).

1. Trichorhina minutissima, n. sp.

2. Trichorhina micros, n. sp.

aa. Dentibus $4+4$ (dentes 1, 3 apice fisso, dentes 2, 4 integri).

3. Trichorhina albida Budde-Lund (5), p. 294, Taf. 17, figs. 5-8.

aqa. Dentibus $3+4$ (dentes 1, 2, 3 apice fisso, dens 4 integer).

4. Trichorhina tomentosa Budde-Lund.

5. Trichorhina quisquiliarum Budde-Lund.

The two first species, Tr. minutissima and Tr. micros, I describe below. Of Tr.albida I have only seen one specimen, described in the cited treatise, and I cannot add any new point, whereas I give below new descriptions of the two species $T r$. tomentosa and $T r$. quisquilianum, which I earlier described and placed in the genus Alloniscus.

Alloniscus papillosus Budde-Lund, Entom. Medd. 1893, p. 193 and Alloniscus ambiguus Budde-Lund, ibid., p. 124, both species from Venezuela, which I referred (Budde-Lund (5), p. 293) to the genus Trichorhina, are in the mouth-parts so different from this genus, that I now place them in a new genus, Gedania, which has several representatives, not yet described, in South America. However I should think that the Platyarthrus simoni Dollfus (Ann. Soc. ent. Fr., lxii., p. 342) from Venezuela is a Trichorhina. 
25. Trichorhina minutissima, n. sp. (Plate 21, figs. 16-24). obtecta.

Superficies squamis minutis et setis minutissimis in marginibus clavatis dense

Oculi parvi ; ocelli numero $6-7$, fortius nigropigmentati.

Antennæ breviores, scapi articuli 2, 3, 4 subæque longi, articulus 5 ceteris paulo longior; flagellum articulo 5 scapi vix brevius; articulo priore duplo vel plus breviore quam articulo altero.

Frons ante marginata; linea marginalis utrinque ante oculos paulum producta; epistoma supra convexum, infra inter antennulas tuberculo semicirculato.

Trunci segmentum 1 margine posteriore fortius curvato, segmentum 2 margine posteriore levius curvato, segmenta 3 et 4 margine posteriore subtransverso, segmenta 5 et 6 margine posteriore in medio leviter incurvo, segmentum 7 margine posteriore in medio fortiter incurvo. Anguli laterales posteriores seginentorum 1, 2, 3, 4 rotundate obtusi, anguli laterales posteriores segmentorum 5,6,7 rotundate subrecti.

Caudæ segmenta $3,4,5$ epimeris mediocribus, segmentorum 3 et 4 acutioribus, segmenti 5 obtusioribus.

Telsum triangulum, lateribus leviter incurvis, apice acuto, epimera segmenti 5 paulum superans.

Uropodum scapus telso brevior, latere exteriore ad longitudinem fisso ; exopoditum plus duplo longius quam scapus, teres, latere exteriore ad longitudinem sulcato; endopoditum satis brevius et multo tenuius quam exopoditum.

Unicolor, albida.

Long. $2 \cdot 5-3 \mathrm{~mm}$. Lat. $1-1.25 \mathrm{~mm}$.

Distribution. Cargados; several specimens were taken in Siren Island during the Sealark Expedition.

26. Trichorhina micros, n. sp. (Plate 21, figs. 25-27).

Superficies squamis clavatis minutissimis sparsius obtecta.

Oculi parvi ; ocelli pauci, numero c. 3-4, ægre pigmentati.

Antennæ...

Frons ante marginata; linea marginalis tenuis in medio leviter producta; frons utrinque in processum obtusum producta; epistoma convexum, supra linea elevata transversa in medio procurva, inter processus laterales frontales ducta, infra inter antennulas tuberculo oblongo parvo, in medio coarctato instructum.

Trunci segmenta marginibus posterioribus et angulis lateralibus eodem modo fere ut in $T r$ minutissima formatis.

Caudæ segmenta 3, 4, 5 epimeris mediocribus, acutioribus, subadpressis.

Telsum triangulum, epimera segmenti 5 paulum superans, quarta parte latius quam longius, lateribus subrectis vel levissime incurvis.

Uropodum scapus latere exteriore subintegro.

SECOND SERIES-ZOOLOGY, VOL. XV. 
Color albidus.

Long. $2.3 \mathrm{~mm}$. Lat. $1 \mathrm{~mm}$.

Distribution. Mauritius; I have only seen one specimen belonging to my collection.

27. Trichorhina tomentosa (Plate 22, figs. 1-5).

Alloniscus tomentosus Budde-Lund, Ent. Med. 1895, p. 126.

Bathytropa thermophila Dollfus, Feuil. Jeun. Nat., s. 3, v. 26, p. 94, text-fig. 2, 1896.

Superficies squamis vel setis clavatis minutissimis dense obtecta; margo posterior capitis et omnium segmentorum trunci caudæque serie squamarum majorum setis minutis interpositis munitus.

Oculi simplices, minuti, ægre pigmentati.

Antennæ breviores, dimidio corpore breviores; scapi articulus 4 paulo longior quam articulus 2 ; flagellum scapi articulo 5 longitudine æquale, articulo priore plus duplo breviore quam articulo altero.

Frons linea marginali squamarum clavatarum subrecta, in medio levissime producta, ab epistomate discreta; processus frontales laterales parvi, rotundati. Epistoma supra cum fronte productum, linea transversa elevata in medio subrecta utrinque sinuate recurva, infra tuberculo rotundate tetragono inter antennulas munitum; clypeus magnus, porrectus.

Trunci segmentum 1 margine posteriore curvato, segmenta 2, 3 margine posteriore subrecto, segmenta 4-7 margine posteriore in medio leviter incurvo; anguli postici laterales segmentorum $1-4$ rotundate obtusi, segmentorum 5,6 subrecti, segmenti 7 acutiores. Segmenta 2,3,4, stria suturali manifesta in femina.

Caudæ segmenta $3,4,5$, epimeris majoribus, triangulis, latere exteriore curvato.

Telsum breve, triangulum, duplo vel plus latius quam longius*, epimera segmenti 5 paululum superans, lateribus subrectis, apice obtuso.

Unicolor, albida vel ex albido grisea.

Long. $3-3.5 \mathrm{~mm}$. Lat. $1.3-1.5 \mathrm{~mm}$.

Distribution. Jamaica at Kingston (1905, C. Gagzo in Mus. Hamburg.). Venezuela (Mus. Kjöbenhavn). Ecuador at "Purnio ob Magdalena" (Bürger in Mus. Göttingen); Paris (Dollfus), Haiti, at Port au Prince (Nepperschmidt in Mus. Hamburg.); Kew Garden, London (Bagnall), Naranjito 9. III. 01 (V. Ortoneda), Guayaquil (F. v. Buchwald, 10. VIII. 03, Mus. Hamburg.).

28. Trichorhina quisquiliarum (Plate 22, fig. 6).

Alloniscus quisquiliarum Budde-Lund, Ent. Med., 1895, p. 125.

Tota superficies densius setis clavatis obtecta ; margo posterior omnium segmentorum serie papillarum minutissimarum ornatus.

* Dollfus describes the telson as "aussi long que large," and fig. 5 in pl. 22 of this Memoir represents it far less than twice as broad as long, with incurved sides. 
Oculi parvi ; ocelli pauci, numero c. 6, quorum solum bini pigmentati.

Antennæ corporis dimidium longitudine subæquantes; scapi articuli tres priores inter se longitudine subæquales, articulus 4 sesqui longior quam articulus 3 ; flagelli articulus prior altero fere triplo brevior.

Processus frontales laterales parvi, obliqui ; epistoma convexum cum fronte paulum productum, infra inter antennulas linea transversa sinuata carinatum.

Trunci segmenta duo priora margine posteriore curvato, segmentum 3 margine posteriore subtransverso.

Caudæ segmenta 3, 4, 5, epimeris brevioribus et latioribus.

Telsum breve triangulum, epimera segmenti 5 vix superans, plus duplo latius quam longius*, lateribus late incurvis*, apice acutiore.

Unicolor, alba.

Long. $2 \cdot 8-3 \mathrm{~mm}$. Lat. $1 \cdot 2-1 \cdot 3 \mathrm{~mm}$.

Distribution. Venezuela.

Alloniscus (Budde-Lund (1), p. 224), Dana, Pr. Ac. Philad. vol. vii., p. 176, 1856.

29. Alloniscus pigmentatus (Plate 22, fig. 7).

Alloniscus pigmentatus Budde-Lund (1), p. 227 ; (5), p. 297, Taf. 15, figs. 23-38.

Chagos Archipelago: Coin, Peros, three; Egmont, eight specimens. Farquhar, four specimens, 30 Sept. 1905. Providence, six specimens. Desroches, one specimen. Aldabra Isl. (H. P. Thomasset). Mahé (Mus. Paris).

This species is very common in Madagascar, and is found in many localities in the East Indies.

I give a figure of the exterior lobe of the first maxillæ, because I have seen that it bears a little appendix not before observed.

30. Alloniscus pallidulus.

Alloniscus pallidulus Budde-Lund (1), p. 228, 1885.

Anomaloniscus ovatus Dollf. (1), p. 187, text-fig. $2 a, b, c, 1893$.

Distribution. Seychelles: Mahé (Anse Royale) sec. Dollfus l.c.

I have not seen specimens of this species from Seychelles, but I have no doubt that the specimens described by Dollfus as Anomaloniscus ovatus belong here.

Alloniscus pallidulus is very common in Madagascar; I have seen specimens taken at "Fort Dauphin," “Vynang du Maramboro," "Tamatave," " bords de la Tarasy," " près du lac d'Anongy," "Androhomana dans grotte," "Baie d'Antongil côte Est" (M. Ch. Alluaud in Mus. Paris), at Isl. Ste. Marie (Professor Voeltzkow in Mus. Berlin), at Tamatave (Herr A. O'Swald in Mus. Hamburg). One specimen taken at Fort Dauphin has an extraordinary size, $19 \mathrm{~mm}$. long by $12 \mathrm{~mm}$. broad.

A third species, Alloniscus brevis Budde-Lund, has been taken at the Comoro Islands (Budde-Lund (5), p. 298, pl. 15, figs. 39, 40); the other species of this genus are indigenous in the East Indies and Australasia.

* Fig. 6 in pl. 22 does not agree with these characters. 
Alloniscus maldivensis Borradaile (Land Crustaceans in Gardiner's Fauna Maldives and Laccadives, vol. i., p. 98, text-figs. 2, $3 a, b, 1901)$ from the Maldive Archipelago is scarcely different from $A l l$. pigmentatus; the description gives no information*.

Genus Setaphora (Budde-Lund (5), p. 290), 1908.

In the quoted paper I have given the provisional characters of this genus which I have separated from the large genus Philoscia.

Of the species of Philoscia, registered in Crust. Isop. Terr. only one species, $P h$. angusticauda Budde-Lund (1), p. 216, from "Borneo," has to be placed in the genus Setaphora. But of species of Philoscia afterwards described the following belong to this genus :

Ph. lubricata Budde-Lund (Ann. Mus. Civ. Gen. 5. 2, v. 14, p. 610), from "Burmah." Ph. comta Budde-Lund (ibid., p. 611), from Burmah.

Ph. cceca Budde-Lund (ibid., p. 611), from Burmah.

Ph. truncatella Budde-Lund (Proc. Zool. Soc. Lond. 1902, p. 379), from "Malay Peninsula."

Ph. incurva Budde-Lund (ibid., p. 380), from "Malay Peninsula."

Ph. suarezi Dollfus (Mém. Soc. Zool. France, v. 8, p. 185), from "Madagascar, East Africa."

Ph. laticeps Bagnall (Ann. and Mag. Nat. Hist., ser. 8, v. 1, p. 429), "Europe," imported? and I dare say also the following:

Ph. truncata Dollfus (Weber, Reise Niederl. Ost. Ind. iv., p. 376), from Celebes and Flores.

Ph. variegata Dollfus (ibid., p. 377), from Celebes.

Ph. weberi Dollfus (ibid., p. 378), from Sumatra.

Ph. cinctella Dollfus (ibid., p. 378), from Celebes.

Ph. sundaica Dollfus (ibid., p. 379), from Sumatra and Java.

Ph. pallida Dollfus (ibid., p. 380), from Java.

Ph. alba Dollfus (ibid., p. 381), from Celebes.

Ph. lifuensis Stebbing (A. Willey's Zool. Results, v., 1900, p. 648).

Besides the three new species described below, I know about twenty undescribed species of the genus Setaphora from different localities, most of them from the East Indies and the Indian islands.

31. Setaphora ovata, n. sp. (Plate 22, figs. 8-13).

Superficies lævis, nitida, vix punctata.

Oculi mediocres, ocelli dense congregati, numero c. 20 .

* The description in fact agrees closely with that given in 1885 without figures by Budde-Lund for his A. pigmentatus, except that the length of that species is stated to be $10 \mathrm{~mm}$., while the longest specimen of $A$. maldivensis measured only $3 \cdot 5 \mathrm{~mm}$. 
Antennæ duas corporis partes longitudine vix æquantes $\left(\frac{8}{14}\right)$; flagellum scapi articulo 5 non longius, articuli subæquilongi.

Caput fere duplo latius quam longius. Frons ante non marginata. Epistoma supra planum, linea transversa solita in medio paululo procurva.

Trunci segmenta 1 et 2 margine postico valde curvato, segmentum 3 margine postico leviter curvato, segmenta 4, 5, 6, 7, margine postico medio sensim magis incurvo. Anguli externi segmenti 1 late rotundati, segmenti 2 rotundate obtusi, segmenti 3 obtusi, segmenti 4 subrecti, segmentorum 5, 6, 7, sensim acutiores. Segmentum 2 pronoto $\frac{1}{4}$ dorsi non superante, ad latera majore, angulos laterales anticos late occupante.

Cauda corpore non abrupte angustior; segmenta longitudine subæqualia, epimeris paulum distantibus.

Telsum breve, triplo latius quam longius, triangulum post rotundatum.

Uropodum scapus latere exteriore ad longitudinem canaliculato, processus endopoditi nonnihil brevior quam scapus.

Color e brunneo violaceus, in dorso sæpe pallide evanescens, maculis albidis conspersis, ad latera macula parva rotunda in trunci segmentis $1-7$, anguli postici epimerorum sæpe perlucentes ; pedes pallidi, antennæ brunneæ, radice flavo.

Long. $7.5 \mathrm{~mm}$. Lat. $3.5 \mathrm{~mm}$.

Distribution. Seychelles, some specimens taken by Dr A. Brauer under palm leaves at Mahé, 2-8 May 1901.

32. Setaphora pallidemaculata, n. sp. (Plate 22, figs. 14-18).

Superficies lævis, nitida, vix punctata.

Oculi mediocres, angulos anticos capitis utrinque occupantes; ocelli dense congregati, numero c. 20.

Antennæ tres quartas partes corporis longitudine æquantes; flagellum articulo 5 scapi longitudine æquale, articulus 1 articulis 2 et 3 subæqualibus simul sumptis fere longior, articulus 3 stylo apicali dimidio articulo longitudine subæquali munitus.

Frons ante non marginata. Epistoma supra in medio carina ad longitudinem ducta leviore, inter margines superiores foraminum antennarum linea tenui elevata transversa in medio leviter procurva ducta.

Trunci segmentum 1 linea collari simplice, ad angulum anticum segmenti utrinque ducta, area articulari capitis solum antica minima; margo posterior valde curvatus, angulis externis late rotundatis ; anguli antici segmenti late rotundate obtusi. Segmentum 2 pronoto $\frac{1}{4}$ dorsi paulum superante, ad latera majore, angulos anticos late occupante; margo posterior leviter curvatus, angulis externis late rotundatis. Segmenta 3 et 4 margine postico subrecto vel leviter curvato, angulis externis late rotundatis. Segmenta 5, 6, 7, margine postico medio post sensim magis incurvo, angulis segmenti 5 obtusis, segmentorum 6 et 7 acutioribus. Stria suturalis epimeri in femina manifesta in segmentis $2,3,4$. 
Caudæ segmentum 1 subobtectum, segmentum 2 breve, segmenta 3, 4, 5 majora; epimera segmenti 3, 4, 5 parva, adpressa, acuta, retroducta.

Telsum breve triangulum, post rotundatum.

Uropodum scapus latere exteriore ad longitudinem canaliculato, processus endopoditi nonnihil brevior quam scapus.

Color e griseo vel e fusco violaceus, maculis et striis parvis dorsalibus albidis; anguli postici trunci segmentorum pellucide albidi ; pedes grisei leviter violaceo picti.

Long. $10-12 \mathrm{~mm}$. Lat. $4-4.5 \mathrm{~mm}$.

Distribution. Seychelles; the Sealark Expedition collected many specimens, chiefly in Mahé, at Montagne Alphonse, $1800 \mathrm{ft}$., the 4 Dec. 1905; Mt. Harrison, $2000 \mathrm{ft}$., Trois frères, $1500 \mathrm{ft} ., 17$ Nov. 1905 ; mountain forest, Cascade, $2000 \mathrm{ft} ., 3$ Dec. 1905 : also in Silhouette 1 specimen and in Praslin 2 specimens. In the museum at Hamburg are many specimens, taken by Dr A. Brauer, 2 to 8 April, 1901.

\section{Setaphora suarezi.}

Philoscia suarezi Dollfus, Mém. Soc. Zool. Fr. v. 8, p. 185, text-fig. 7, 1895.

Setaphora suarezi Budde-Lund (5), p. 291, Taf. 16, fig. 6-14, (7), p. 15.

Several specimens from Coetivy and Farquhar. This species is common in East Africa, in the Comoro Islands and in North Madagascar.

34. Setaphora pilosa, n. sp. (Plate 22, figs. 19-23).

Superficies sparse, in segmentis caudæ densius, setis minutissimis obtecta.

Oculi magni ; ocelli numero circiter $17-18$.

Antennæ longæ, duas partes corporis longitudine superantes; flagellum articulo 5 scapi paululo longius, articulus 1 longissimus, articuli 2 et 3 æquilongi, articulus 3 seta apicali longa.

Frons ante non marginata; lobi laterales parvi, adpressi, rotundate ovales. Epistoma supra planum, infra linea elevata transversa, subrecta.

Trunci segmentum 1 margine posteriore valde curvato, segmenta 2, 3, 4, margine posteriore leviter curvato, subrecto, segmenta 5, 6 leviter segmentum 7 fortiter margine postico medio incurvo; anguli postici segmentorum $1-5$ obtusi, rotundati, anguli postici segmentorum $6-7$ subrecti.

Caudæ segmenta $3,4,5$, epimeris minimis, adpressis, acutissimis.

Telsum breve triangulum, lateribus leviter incurvis, apice late rotundato.

Color e fusco violaceus, maculis striis parvis punctis albescentibus crebratus, in epimeris segmentorum $2,3,4,5$, trunci macula oblonga albida utrinque posita, in epimeris ceterorum segmentorum macula evanida; anguli postici trunci segmentorum maxime posteriorum perlucente albidi; antennæ fuscæ, flagello flavo.

Long. $5 \mathrm{~mm}$. Lat. $2 \mathrm{~mm}$.

Distribution. Chagos Archipelago: many specimens from Salomon; 3 specimens from Diego Garcia; 6 specimens from Egmont. 
Genus Apriloscia (Budde-Lund (5), p. 291), 1908.

35. Aphiloscia annulicornis.

Philoscia annulicornis Budde-Lund (1), p. 211, 1885.

" " Dollf., Mém. Soc. Zool. Fr., v. 8, p. 184, text-fig. 6, 1895.

Aphiloscia ," Budde-Lund (5), p. 291 ; Taf. 16, fig. 15-31, 1908.

Seychelles Archipelago: Mahé, mountain forest, Cascade, one large specimen, $9.5 \mathrm{~mm}$. long, $4.5 \mathrm{~mm}$. broad. Farquhar, 30 Sept. 1905, several small specimens.

This species seems to be common in Madagascar and in the Comoro Islands; it is also found in Mauritius and Réunion.

\section{Subfamilia Olibrininae.}

Antennæ longissimæ, flagello longo, multiarticulato (10-15).

Maxillæ prioris paris lamina exterior longa, angusta, margine posteriore nudo, apice dentibus c. duodecim, omnibus integris, simul appendice longa, mobili; lamina interior apice dense crinito penicillis duobus minus manifesto discretis instructa.

Maxillæ alterius paris lamina angusta indivisa, apice integro, dense crinito.

Maxillipedum articulus labialis angustus, margine exteriore et interiore ad apicem pilis longis instructo; palpus magnus, latus, 2-articulatus, articulus 1 brevis spinis solitis duabus minutis instructus, articulus 2 permagnus, latus, margine interiore ad apicem undulate producto cum margine exteriore crinibus longis instructo; mala palpo multo brevior, ad apicem angustata.

Exopoditum pleopodum trachea destitutum.

Olibrinus nov. gen.

Having rather hurriedly to give a report of the collection of terrestrial Isopods from the Malayan Peninsula gathered by the Skeat Expedition, I had no opportunity to make a profound examination of the few specimens of the below-mentioned species; on account of its habitus and the multi-articulated flagellum of the antennæ I placed the species in the genus Trichoniscus. I now have had a somewhat larger number of specimens for examination, and, as it seems, of three different though very nearly allied species. Olibrinus has to be placed in the family Oniscoida, even though not only by the antennæ but also by the structure of the trophi it makes a transition to the family Ligiidce. I have, however, considered it necessary to distinguish it as a subfamily.

36. Olibrinus antennatus (Plate 22, figs. 24-26).

Trichoniscus antennatus Budde-Lund in Proc. Zool. Soc. London, 1902, p. 379.

Superficies lævis, nitida.

Oculi minores; ocelli 8-10.

Antennæ perlongæ, gracillimæ, corpus longitudine paulum superantes; scapi articulus 1 brevis; articuli 2 et 3 breviores, subæquales; articulus 4 fere duplo longior quam 
articulus 3 ; articulus 5 paulo, fere quarta parte longior quam articuli 3 et 4 simul sumpti; flagellum paulo longius quam scapi articulus 5, 15-articulatum, articulo primo ceteris longiore.

Trunci segmenta quattuor priora margine posteriore subrecto vel leviter curvato, angulis posticis rotundate obtusis; segmenta 5 et 6 margine posteriore in medio leviter incurvo; segmentum 7 margine posteriore in medio fortiter incurvo; anguli postici segmenti 5 subrecti, segmentorum 6 et 7 acutiores. Segmentum 2 pronoto quarta parte dorsi vix longiore, utrinque intra angulos laterales desinente.

Caudæ segmenta $1-\mathbf{j}$ post sensim majora, epimeris minimis, adpressis, inflexis.

Telsum breve, medio leviter rotundate triangulo producto.

Uropodes producti, longi, tertiam corporis partem longitudine paulum superantes; scapus teres, oblongus, plus duplo longior quam latior; exopoditum elongate conoidale, multo, fere duplo, longius quam scapus, apice setis brevibus instructum; endopoditum processui brevi scapi insertum, quam scapus paulo brevius, apice tamen scapum superante.

Subunicolor e fusco violaceus.

Long. 5-6 mm. Lat. $2-2 \cdot 3 \mathrm{~mm}$.

Distribution. Malay Peninsula.

37. Olibrinus pigmentatus, n. sp.

Oculi minores, ocelli numero 11.

Antennæ perlongæ, corpore vix breviores. Flagellum articulo 5 scapi longius, 13-articulatum; articulus primus ceteris paulo longior.

Frons ante marginata, linea marginalis utrinque decurrens, lobos minores amplectens. Fossæ antennarum permagnæ, maximam partem inferiorem epistomatis occupantes. Antennulæ sese fere attingentes, spatium inter fossas antennarum occupantes. Clypeus magnus leviter fornicatus; labrum magnum porrectum. Os porrectum.

Trunci segmenta 1, 2, 3 margine posteriore leviter curvato, utrinque vix incurvo; segmentum 4 margine posteriore subrecto; segmenta 5, 6, 7 margine posteriore in medio post sensim magis incurvo; anguli posteriores segmentorum 1, 2, 3, 4 rotundate obtusi, 5 subrecti, 6,7 acuti.

Caudæ segmenta 5 priora subæque longa, epimeris omnibus minutis, adpressis. Segmenta 1, 2 brevia, 3, 4, 5 longiora, epimeris perparvis acutis.

Pleopodes trachea nulla muniti.

Telsum brevissimum, medio anguste rotundate paulum producto.

Uropodes longi, producti, tertiam corporis partem longitudine subæquantes ; scapus angustus, processu endopoditi brevi; endopoditum gracile scapum paulum superans; exopoditum longum dimidio longius quam scapus, gracile, teres.

Color albidus, punctis nigris minutissimis pigmentatus.

Long. $6 \mathrm{~mm}$. Lat. $2 \cdot 2 \mathrm{~mm}$.

Distribution. Chagos Archipelago: Salomon, one specimen; Coin, Peros, four specimens. 
38. Olibrinus olivaceus, n. sp.

Oculi minores, ocelli majores, c. 10.

Antennæ longæ, gracillimæ, paulo $\left(\frac{7}{8}\right)$ breviores quam corpus; articulus 1 scapi brevissimus; articuli 2 et 3 breves subæquales; articulus 4 plus duplo longior quam articulus 3 ; articulus 5 vix longior quam articuli 3 et 4 simul sumpti; flagellum fere quarta parte longius quam articulus 5 scapi, 10-articulatum, articulo primo longiore quam ceteris.

Mandibula dextra penicillo altero fixo, altero libero.

Frons ante marginata; linea marginalis medio subrecto, utrinque in lobos laterales mediocres obtusos, deflexos producta.

Trunci segmenta 1, 2, 3 margine posteriore leviter curvato vel subrecto; segmentum 4 margine posteriore recto; segmenta 5, 6 margine posteriore in medio incurvo; segmentum 7 margine posteriore in medio fortiter incurvo ; anguli posteriores segmentorum 1, 2, 3, 4, late rotundate obtusi, segmenti 5 rotundati subrecti, segmentorum 6,7 acuti. Pronotum segmenti \& quartæ parti dorsi longitudine æquale.

Caudæ segmenta 3, 4, 5 post sensim longiora, epimeris parvis, adpressis, acutis, retroductis.

Telsum breve, medio semicirculatim producto.

Subunicolor e griseo olivaceus.

Long. $8 \mathrm{~mm}$. Lat. $2.5 \mathrm{~mm}$.

Distribution. A few specimens were taken at Djibouti by Prof. H. Coutière in the year 1897 (Mus. Paris).

Familia Tylidæ (Tylides, Budde-Lund (1), p. 272), 1885.

39. Tylos minor.

Tylos minor Dollf. (1), p. 189, text-fig. $4 a-e, 1893$.

Seychelles, sec. Dollf: "Mahé, digue, plage, sous Algues" (Ch. Alluaud).

Familia Ligiidæ (Ligiae, Budde-Lund (1), p. 242), 1885.

40. Ligia exotica.

Ligia exotica Roux (Crustacés de la Méditerranée et de son littoral...Marseille 1828, Livr. 3, Tb. 13, fig. 9).

" , Budde-Lund (1), p. 266.

Seychelles, sec. Dollf.: “Plage de l'île Ronde, rochers près de la mer” (Ch. Alluaud). 


\section{LITERATURE.}

The papers most frequently quoted with abbreviated titles are the following :

Budde-Lund. (1) Crustacea Isopoda Terrestria, Hauniae, 1885.

(2) A Revision of Crustacea Isopoda Terrestria: I. Eubelum, Kjöbenhavn, 1899 (5 Tabb.); II. Spherilloninæ; III. Armadillo, Kjöbenhavn, 1904 (5 Tabb.).

(3) Die Land-Isopoden der Deutschen Südpolar-Expedition, 1901-1903, Ix. Zoologie i. Berlin, 1906. (Mit 2 Taf.)

(4) Terrestrial Isopoda from Egypt, in: Results of the Swedish Zoological Expedition to Egypt and the White Nile, 1901, under the direction of L. A. Jägerskiöld. Nr. 26, A. Upsala, 1908. (With 1 Tab.)

(5) Isopoda von Madagascar und Ostafrika, in: Voeltzkow, Reise in Ostafrika in den Jahren 1903-1904, ii. Stuttgart, 1908. (Mit 7 Taf.)

(6) Land-Isopoden, in: L. Schultze, Forschungsreise im westlichen und zentralen Südafrika, ausgeführt in den Jahren 1903-1905 (Denkschr. d. medi.naturwiss. Ges. xiv. Jena 1909). (Mit 3 Taf.)

(7) Crustacea. 2 Isopoda, in: Wissenschaftliche Ergebnisse d. Schwedischen zoologischen Expedition nach dem Kilimandjaro, dem Meru und den umgebenden Massaisteppen Deutsch-Ostafrikas, 1905-1906. Stockholm, 1910. (With 2 Plates.)

Dollfus, AD. (1) Crustacés Isopodes terrestres, in: Voyage de M. Charles Alluaud aux Iles Séchelles. (Bull. d. l. Soc. Zool. de France, t. xviii. p. 186. Paris, 1893.) (With text figures.)

(2) Isopodes terrestres recueillis à Diégo-Suarez, à Tamatave et à la Réunion, in: Mission de M. Ch. Alluaud dans le territoire de Diégo-Suarez (MadagascarNord), Avril-Août, 1893. (Mém. d. l. Soc. Zool. de France, t. viii. p. 180. Paris, 1895.) (With text figures.) 


\section{EXPLANATION OF PLATES 20-22.}

\section{Plate 20.}

Fig. 1. Pseudophiloscia lateralis, n. sp. Distal portion of the right mandible, from below. $\times 135$.

Fig. 2.

Fig. 3.

Fig. 4.

Fig. 5.

Fig. 6.

Fig. 7.

Fig. 8.

Fig. 9.

Fig. 10.

Fig. 11.

Fig. 12.

Fig. 13.

Fig. 14.

Fig. 15.

Fig. 16.

Fig. 17.

Fig. 18.

Fig. 19.

Fig. 20.

Fig. 21.

Fig. 22.

Fig. 23.

Fig. 24 .

Fig. 25.

Fig. 26.

\section{"}

$"$

,

"

,"

,

,
,

",

,

"

", angustissima, n. sp.

,

, ,

brevicornis, n. sp.

"

"

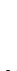

"

,

(2)

Distal portion of the left mandible, from below. $\times 135$.

Distal portion of the exopodite of right 1st maxilla. $\times 135$.

Distal portion of the endopodite of the same maxilla. $\times 135$.

Distal portion of the left pes maxillaris. $\times 75$.

Right uropod. $\times 16$.

Distal portion of the left mandible, from above. $\times 135$.

Distal portion of the exopodite of the left 1st maxilla. $\times 250$.

Distal portion of the left pes maxillaris. $\times 150$.

Distal portion of the right mandible, from below. $\times 150$.

Distal portion of the left mandible, from below. $\times 150$.

Distal portion of the exopodite of the left 1st maxilla. $\times 135$.

Distal portion of the right pes maxillaris. $\times 150$.

Left uropod, from the outer side. $\times 50$.

Left uropod, from below. $\times 50$.

Mahehia maculata, n. sp. The head, from above. $\times 10$.

" " Right part of the first segment, from above. $\times 4$.

" " " Right part of the second segment, from above. $\times 4$.

$"$ " " $"$ The right antenna. $\times 10$.

$" \quad " \quad$ " $" \quad$ Distal portion of the right mandible, from below. $\times 35$.

$" \quad " \quad$ The apex of the seta of the right mandible. $\times 135$.

$" \quad " \quad$ Distal portion of the exopodite of the left 1st maxilla. $\times 60$.

" " " Distal portion of the right pes maxillaris. $\times 60$.

$" \quad " \quad$ Right uropod of a female. $\times 20$.

laticauda, n. sp. Dorsal aspect. $\times 4$.

Plate 21.

Fig. 1. Mahehia laticauda, n. sp. Distal portion of the left mandible, from below. $\times 60$.

Fig. 2. " " "

Fig. 3. " " "

Fig. $4 . \quad, \quad, \quad$,

Fig. 5. " " "

Fig. 6. " ", ",

Fig. 7. " bicornis, n. sp.

Fig. 8. " " ,

Fig. 9. " " " "

Fig. $10 . \quad$ " ,

Fig. $11 ., "$,
Distal portion of the left mandible, from above. $\times 75$.

1st appendix of the endopodite (lacinia mobilis) of the left mandible, from above. $\times 75$.

Distal portion of the left pes maxillaris. $\times 15$.

7 th leg of the male. $\times 12$.

The apex of the same leg. $\times 135$.

The head, from above. $\times 12$.

Distal portion of the right mandible, from below. $\times 75$.

Distal portion of the exopodite of the left 1st maxilla. $\times 75$.

Posterior part of the cauda. $\times 4$.

Left uropod of the male. $\times 20$. 
Fig. 12. Bethalus simplex Dollfus. Distal portion of the right mandible, from above. $\times 100$.

Fig. 13. " " " Distal portion of the left mandible, from below. $\times 100$.

Fig. 14. " " " $", \quad$ Distal portion of the exopodite of the right 1st maxilla. $\times 200$.

Fig. 15. " " " Distal portion of the left pes maxillaris. $\times 100$.

Fig. 16. Trichorhina minutissima, n. sp. The head, from above. $\times 25$.

Fig. 17. " " " Distal portion of the right mandible, from below. $\times 250$.

Fig. 18. " " " Distal portion of the left mandible, from below. $\times 250$.

Fig. 19. " " " " Distal portion of the exopodite of the left 1st maxilla. $\times 450$.

Fig. 20. " " " " The apex of the endopodite of the right 1st maxilla. $\times 450$.

Fig. 21. " " " Distal portion of the left pes maxillaris. $\times 135$.

Fig. 22. " " " " " "

Fig. 23. " " " Posterior part of the cauda, from above. $\times 30$.

Fig. 24. $" \quad " \quad$ Right uropod. $\times 75$.

Fig. 25. " micros, n. sp. Distal portion of the exopodite of left 1st maxilla. $\times 450$.

Fig. 26. " " " Distal portion of the endopodite of the same maxilla. $\times 350$.

Fig. 27. " " " Distal portion of the right pes maxillaris. $\times 250$.

\section{Plate 22.}

Fig. 1. Trichorhina tomentosa Budde-Lund. Distal portion of the right mandible, from below. $\times 250$.

Fig. 2. " " Distal portion of the left mandible, from above. $\times 300$.

Fig. 3. " " " " Distal portion of the exopodite of the left 1st maxilla. $\times 350$.

Fig. 4. " " " Distal portion of the left pes maxillaris. $\times 250$.

Fig. 5. " " " Posterior part of the tail. $\times 25$.

Fig. 6. " quisquiliarum Budde-Lund. Posterior part of the tail. $\times 50$.

Fig. 7. Alloniscus pigmentatus Budde-Lund. Distal portion of the exopodite of right 1st maxilla. $\times 135$.

Fig. 8. Setaphora ovata, n. sp. Distal portion of the right mandible, from above. $\times 135$.

Fig. 9. " " " Distal portion of the left mandible, from above. $\times 135$.

Fig. 10. " " " Distal portion of the exopodite of the left 1st maxilla. $\times 250$.

Fig. 11. " " " Distal portion of the endopodite of the same. $\times 170$.

Fig. 12. " " " Distal portion of the left pes maxillaris. $\times 135$.

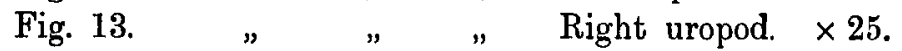

Fig. 14. " pallidemaculata, n. sp. Distal portion of the left mandible, from below. $\times 75$.

Fig. 15. " " " " Distal portion of the exopodite of the left 1st maxilla. $\times 135$.

Fig. 16. " " " " Distal portion of the endopodite of the left 1st maxilla.

Fig. 17. $\quad, \quad$ " $\quad$ Distal portion of the left pes maxillaris. $\times 135$.

Fig. 18. " " " $"$ Right uropod. $\times 15$.

Fig. 19. " pilosa, n. sp. Distal portion of the exopodite of the right 1st maxilla. $\times 250$.

Fig. 20. " " " Distal portion of the endopodite of the same. $\times 250$.

Fig. 21. " " " Distal portion of the left pes maxillaris. $\times 150$.

Fig. 22. " " " Left lst leg of male. $\times 70$.

Fig. 23. " " " Right uropod. $\times 25$.

Fig. 24. Olibrinus antennatus Budde-Lund. Antenna. $\times 15$.

Fig. 25. " " $\quad$ Distal portion of the exopodite of the left 1st maxilla. $\times 250$.

Fig. 26. " " $" \quad$ Left uropod. $\times 30$. 
Percy SLaden TRUSt Expedition. (BUDDE- LUND)
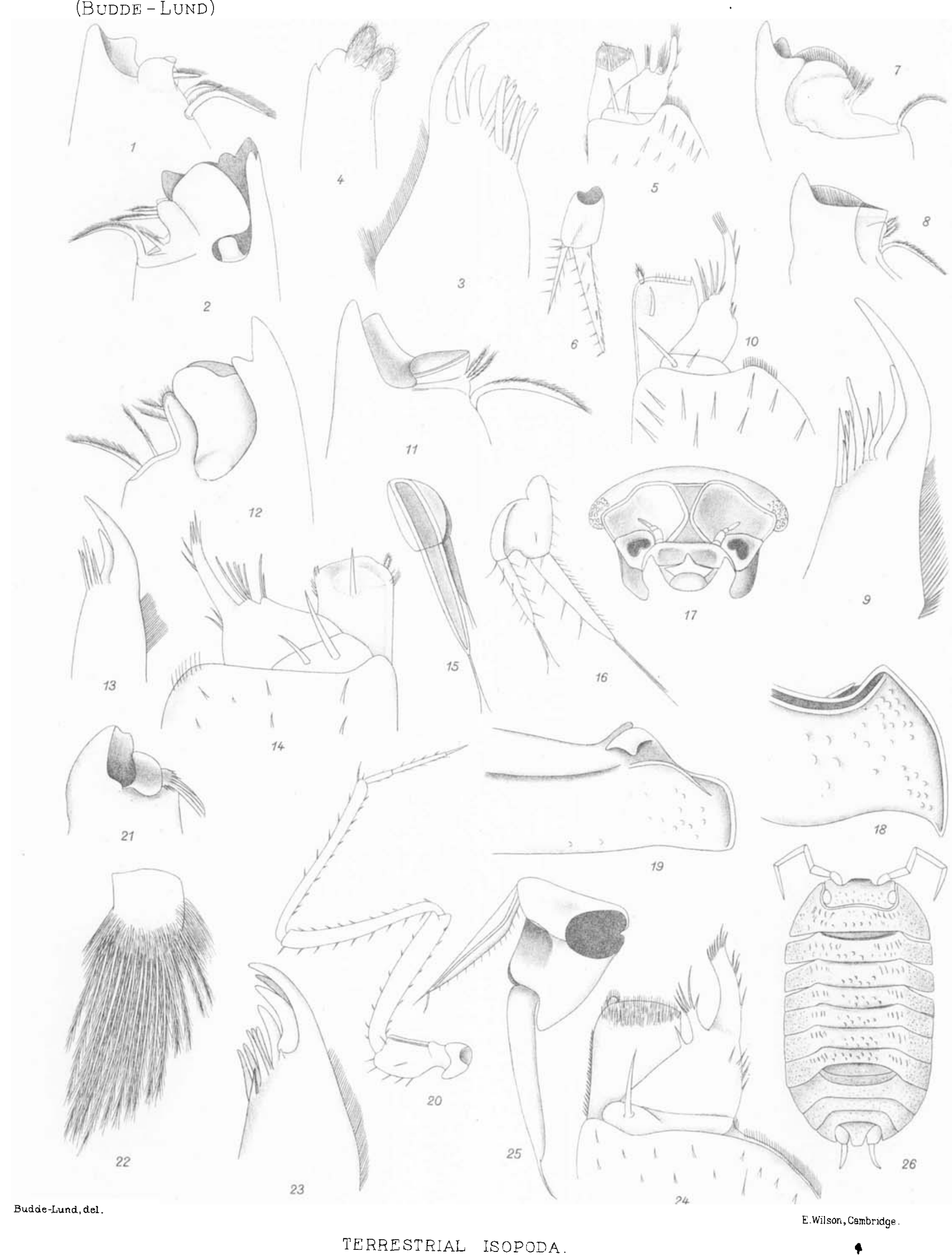

Trans. Linn. Soc., Ser. 2.ZOOL Vol.XV PL 20. 
PERCY SLADEN TRUST EXPEDITION.

Trans. Linn. Soc., Ser. 2.Zool.Vol.XV. Pl. 21. (BUDDE - LUND)
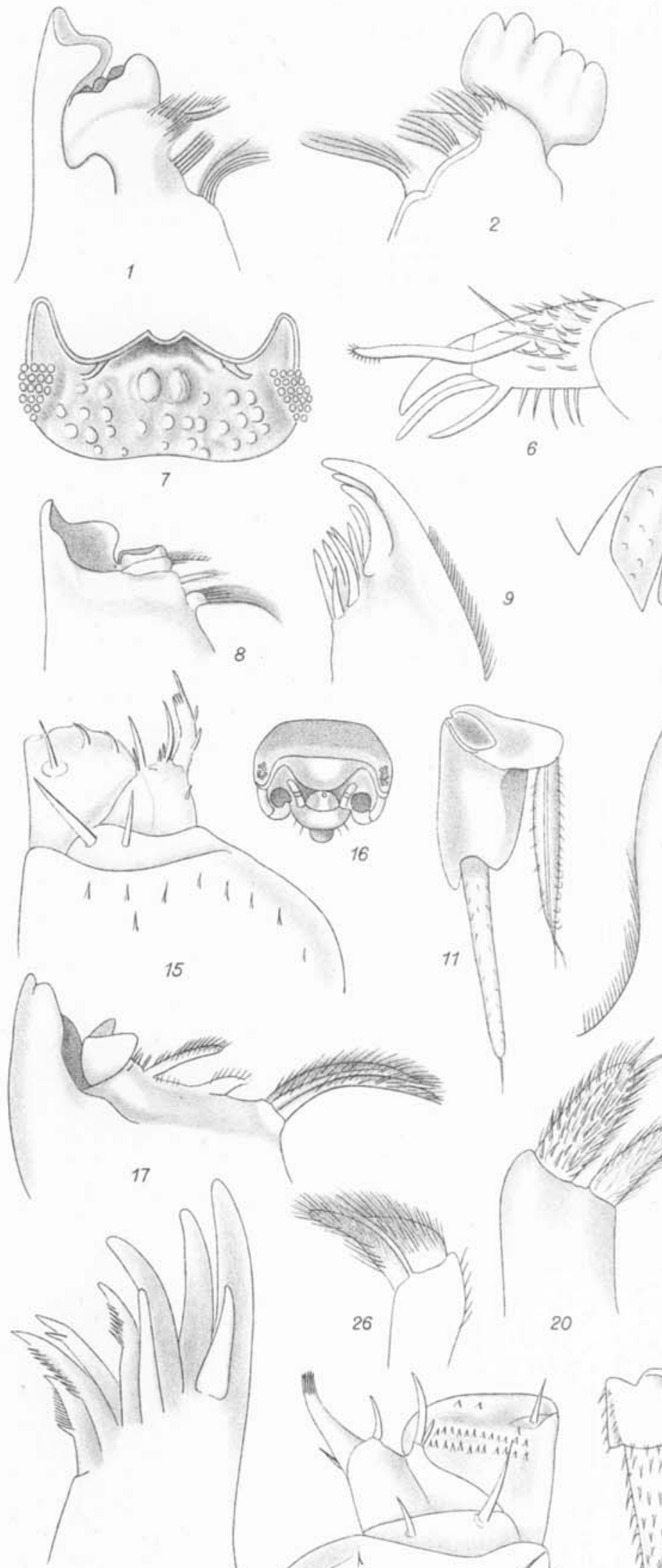

Budde-Lund, del.

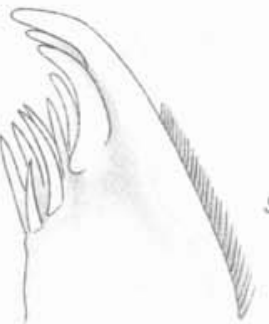

20

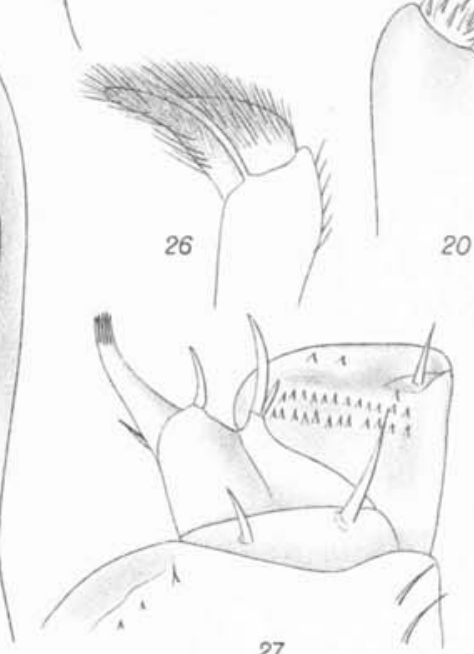

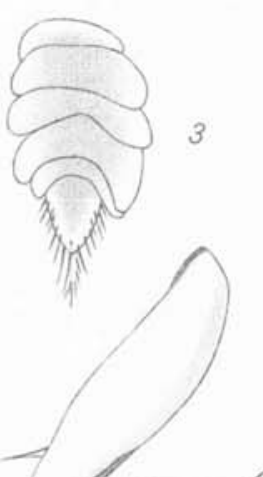
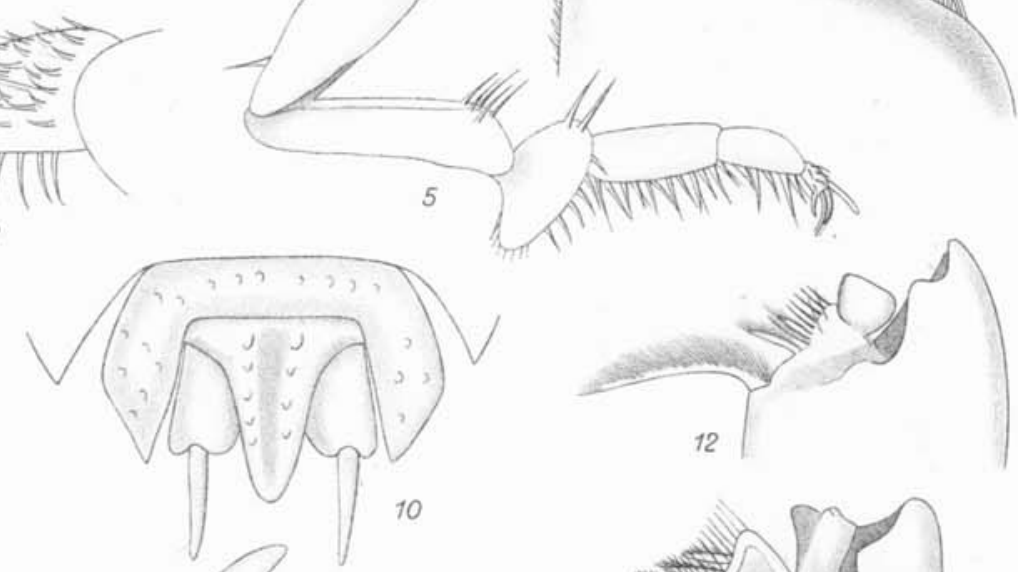

त्ञाजायागणा?
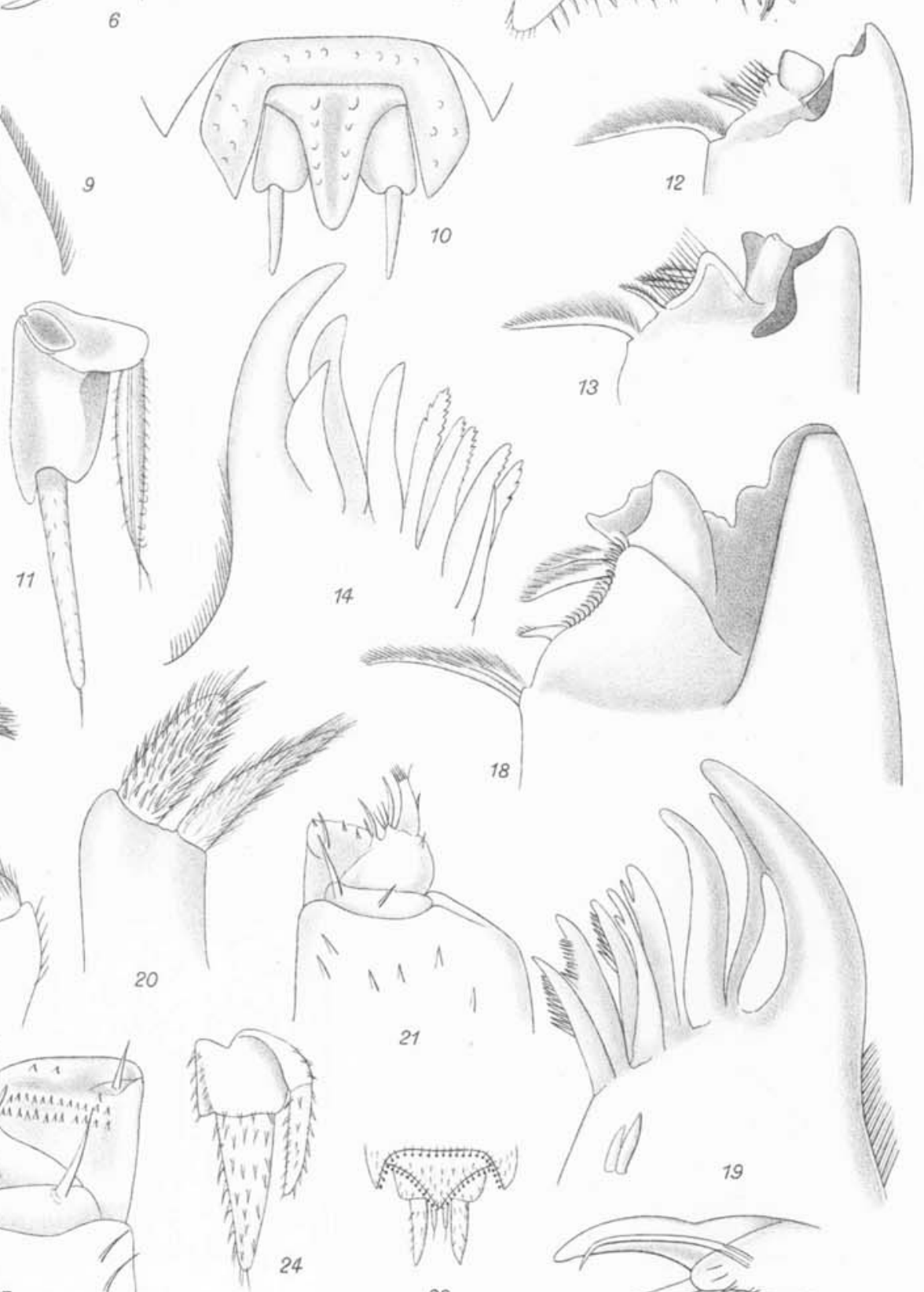

24

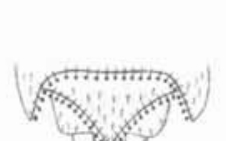

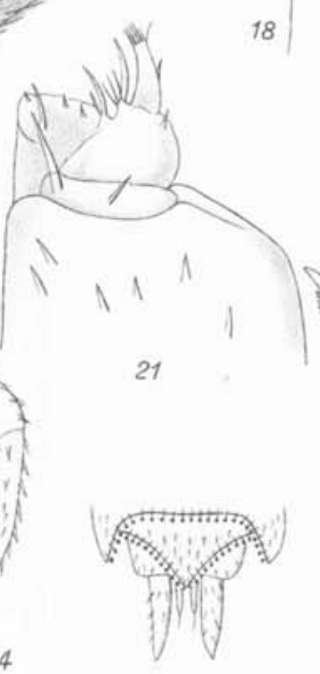

23
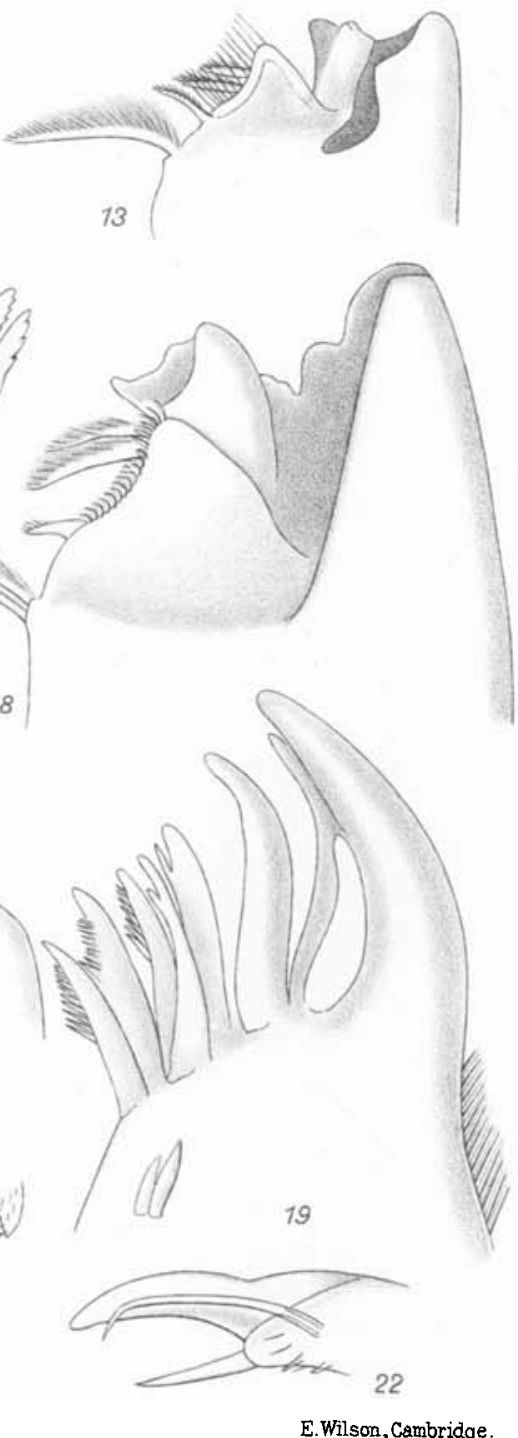

TERRESTRIAL ISOPODA 
Percy Sladen Trust EXPEdition.

Trans. Linn. Soc. Ser. 2.Zool. Vol.XV.Pu. 22.

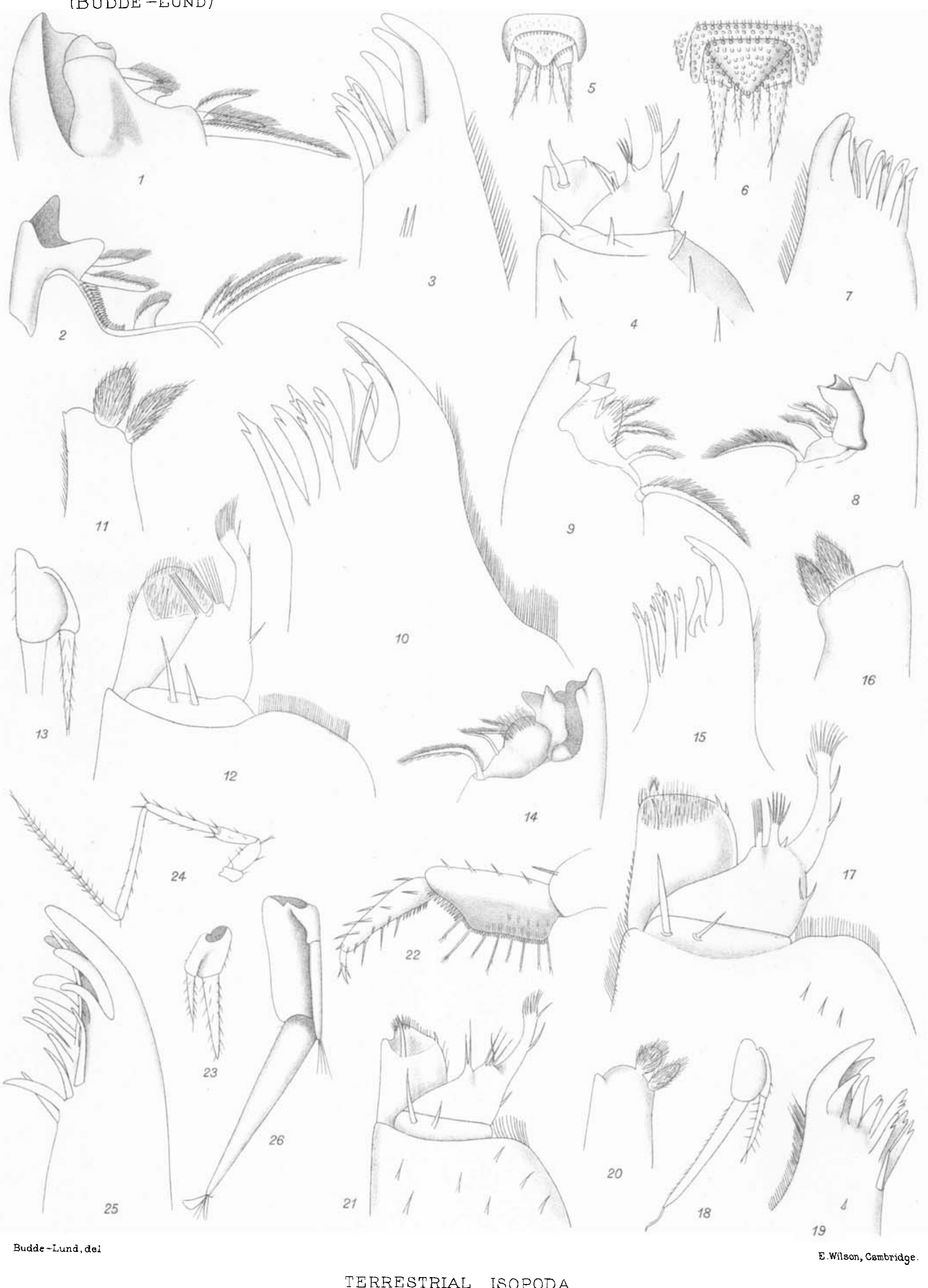

